\title{
Blood Pressure and the Risk of Death From Non-cardiovascular Diseases: A Population-based Cohort Study of Korean Adults
}

\author{
Jeoungbin Choi ${ }^{1,2}$, Jieun Jang ${ }^{1,2}$, Yoonsuk An ${ }^{1,3}$, Sue K. Park ${ }^{1,2,3}$ \\ ${ }^{1}$ Department of Preventive Medicine, Seoul National University College of Medicine, Seoul, Korea; '2Department of Biomedical Science, Seoul \\ National University Graduate School, Seoul, Korea; ${ }^{3}$ Cancer Research Institute, Seoul National University, Seoul, Korea
}

Objectives: The objective of this study was to assess the relationship between systolic and diastolic blood pressure (SBP, DBP) and the risk of death from specific causes other than cardiovascular diseases.

Methods: We calculated the risk of specific death by SBP and DBP categories for 506508 health examinees in 2002-2003 using hazard ratios (HRs) and 95\% confidence intervals (Cls) in a Cox proportional hazards model.

Results: Compared to normal levels (SBP $<120$ or DBP $<90 \mathrm{mmHg}$ ), stage I systolic and diastolic hypertension (SBP 140-159, DBP 85$89 \mathrm{mmHg}$, respectively) were associated with an increased risk of death from diabetes mellitus, alcoholic liver disease, and renal failure (HR, 1.83; $95 \% \mathrm{Cl}, 1.51$ to $2.22 ; \mathrm{HR}, 1.24 ; 95 \% \mathrm{Cl}, 1.06$ to $1.46 ; \mathrm{HR}, 2.30 ; 95 \% \mathrm{Cl}, 1.64$ to $3.21 ; \mathrm{HR}, 1.67 ; 95 \% \mathrm{Cl}, 1.27$ to $2.20 ; \mathrm{HR}, 1.99$; $95 \% \mathrm{Cl}, 1.41$ to $2.81 ; \mathrm{HR}, 1.31 ; 95 \% \mathrm{Cl}, 0.99$ to 1.73 , respectively), but a decreased risk of death from intestinal pneumonia (HR, 0.64; $95 \% \mathrm{Cl}, 0.42$ to $0.98 ; \mathrm{HR}, 0.59 ; 95 \% \mathrm{Cl}, 0.39$ to 0.91 ). Only stage II systolic hypertension (SBP $\geq 160 \mathrm{mmHg}$ ) was associated with an increased risk of death from pneumonia, liver cirrhosis, and intestinal ischemia (HR, 1.54; $95 \% \mathrm{Cl}, 1.19$ to $1.98 ; \mathrm{HR}, 1.46 ; 95 \% \mathrm{Cl}, 1.00$ to 2.15; HR, 3.77; $95 \% \mathrm{Cl}, 1.24$ to 11.40 , respectively), and stage I and II diastolic hypertension (SBP 140-159 and $\geq 160 \mathrm{mmHg}$ ) were associated with an increased risk of death from intestinal ischemia $(\mathrm{HR}, 3.07 ; 95 \% \mathrm{Cl}, 1.27$ to $7.38 ; \mathrm{HR}, 4.39 ; 95 \% \mathrm{Cl}, 1.62$ to 11.88 , respectively).

Conclusions: An increase in blood pressure levels may alter the risk of death from certain causes other than cardiovascular diseases, a well-known outcome of hypertension, although the mechanism of these associations is not well documented.

Key words: Hypertension, Cohort studies, Korean population, Blood pressure

\section{INTRODUCTION}

Received: September 7, 2018 Accepted: October 15, 2018

Corresponding author: Sue K. Park, MD, PhD

Department of Preventive Medicine, Seoul National University College of Medicine, 103 Daehak-ro, Jongno-gu, Seoul 03080, Korea

E-mail: suepark@snu.ac.kr

This is an Open Access article distributed under the terms of the Creative Commons Attribution Non-Commercial License (http://creativecommons.org/licenses/by$\mathrm{nc} / 4.0 /$ ) which permits unrestricted non-commercial use, distribution, and reproduction in any medium, provided the original work is properly cited.
Hypertension is defined as a sustained elevation of systemic arterial blood pressure (BP). In last 16 years, the mean BP of the general Korean population has decreased, potentially leading to changes in health outcomes [1]. Hypertension is a well-verified risk factor of mortality from cardiovascular disease and heart failure [2,3]. In contrast, little has been reported about the relationship of hypertension with major causes of death other than cardiovascular and cerebrovascular mortality 
in Korea or in other countries throughout the world.

Arterial hypertension is known to damage the vascular system, which extends to every organ and all tissues [4], meaning that some organs could share the same mechanism of damage as found in atherosclerosis. The heart, brain, eye, kidney, and arterial aneurysms are generally considered to be sensitive to damage from hypertension, and previous studies have examined the effects of arterial hypertension on these organs [5]. However, few studies have been conducted on other organs that may be affected by arterial hypertension, so the impact of arterial hypertension on other organs remains largely unclear.

The purpose of this study was to investigate the risk of death from specific causes other than well-known causes of death in subjects with hypertension compared to subjects with low BP using Korean large-scale health screening data.

\section{METHODS}

\section{Data Collection and Study Population Selection}

We used data from the National Health Insurance ServiceHealth Screening Cohort (NHIS-HEALS), a large-scale retrospective cohort $(n=515000)$ comprising a $10 \%$ random sample of all health examinees aged 40-70 years who participated in the National Health Insurance health screening program in 20022003 [6]. Information on systolic and diastolic BP (SBP and DBP), anthropometric indices (e.g., height, weight, and body mass index [BMI]), laboratory blood and urine test results, and self-reported questionnaire data were collected in the baseline year of 2002-2003, and the data were recollected biennially (annually for non-white collar workers). We excluded participants without information about their BP $(n=336)$ and those who died within 2 years of cohort enrollment $(n=4228)$. To account for the effects of BMI, a major confounder of the association between BP and death risk, we additionally excluded those who were extremely underweight $\left(\mathrm{BMI}<15 \mathrm{~kg} / \mathrm{m}^{2}\right)$, extremely obese $\left(\mathrm{BMI}>50 \mathrm{~kg} / \mathrm{m}^{2}\right)$, or did not have BMI information $(n=637)$. Furthermore, participants with very high hemoglobin levels ( $>18.3 \mathrm{mg} / \mathrm{dL})(\mathrm{n}=310)$ were also excluded because the elevated risk of death due to high hemoglobin levels could be misinterpreted as a result of hypertension, since hemoglobin levels are positively correlated with BP and higher hemoglobin levels have shown an association with mortality $[7,8]$.

Finally, a total of 506508 subjects were included as the study population. Within this population, a total of 33215 all- cause deaths occurred during up to 11 years of follow-up.

This study was reviewed and approved by the institutional review board (IRB) of Seoul National University Hospital (IRB no. 1806-149-953).

\section{Outcome Variables}

Information on death (date and cause of death) from Statistics Korea was individually linked using unique personal identification numbers [6]. The main outcomes for this study were all-cause death and specific death, except for (1) disease codes including well-known causes of death related to hypertension: cerebrovascular and cardiovascular diseases (I codes); (2) disease codes including causes of death that are less well-known to be associated with hypertension: neoplasms (all C and D codes), non-disease causes of death ( $S, T, V, Y$ codes), and causes of death related to pregnancy, the prenatal period, and congenital abnormalities ( $\mathrm{O}, \mathrm{P}, \mathrm{R}$ codes); and (3) cases affected by the masking policy of the NHIS-HEALS public open data: mental, behavioral and neurodevelopmental disorders (F codes), as defined by the International Classification of Diseases, 10th Revision (ICD-10). We included individual causes of death for which at least 40 deaths were observed for a single ICD-10 disease code in the study population: (1) tuberculosis (A15-A19; $n=261$ ), hepatitis (B15-B19; $n=148$ ); (2) diabetes mellitus ( $D M$; E11-E14; $n=1203)$, including non-insulin-dependent DM (E11; $\mathrm{n}=793) ;$ (3) Parkinson disease (G20; $\mathrm{n}=287)$, Alzheimer disease (G30; $n=195) ;(4)$ pneumonia (J12-J19; $n=783)$, interstitial pneumonia (J84; $n=187$ ), chronic obstructive pulmonary disease (J43-J44; $n=130)$, aspiration pneumonia (J69; $n=857) ;(5)$ alcoholic liver disease (ALD; $K 70 ; n=388$ ), liver cirrhosis (LC; K74; $\mathrm{n}=357$ ), intestinal ischemia (vascular disorders of intestine; $\mathrm{K} 55 ; \mathrm{n}=40)$, paralytic ileus except hernia (K56; $\mathrm{n}=41)$, pancreatitis (K85-86; $n=49)$, including acute pancreatitis (K85; $n=40)$; (6) renal failure (RF; N17-N19; $n=378$ ), including chronic renal failure (CRF; N18; $n=294)$, and acute renal failure (ARF; N17; $\mathrm{n}=48$ ) (Table S1). The follow-up period of each individual was defined as from the day of study enrollment to the date of death (if applicable) or December 31, 2013.

\section{Exposure Variables}

All participants underwent medical examinations at local hospitals. A standard sphygmomanometer was used to measure BP in a seated position, and the SBP and DBP were measured as the first and last Korotkoff sounds, respectively [6]. We used SBP and DBP data measured on the day of study en- 


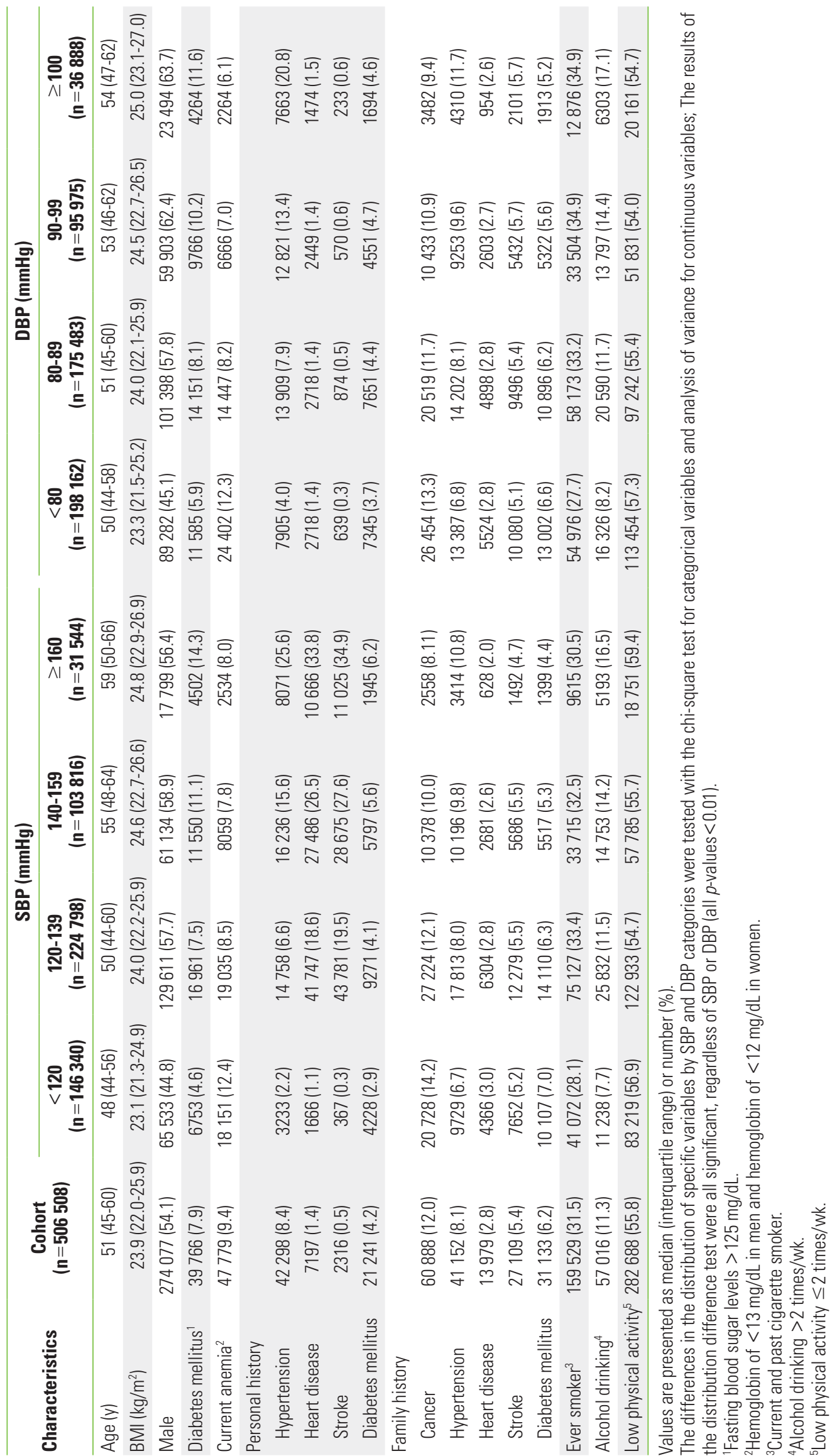



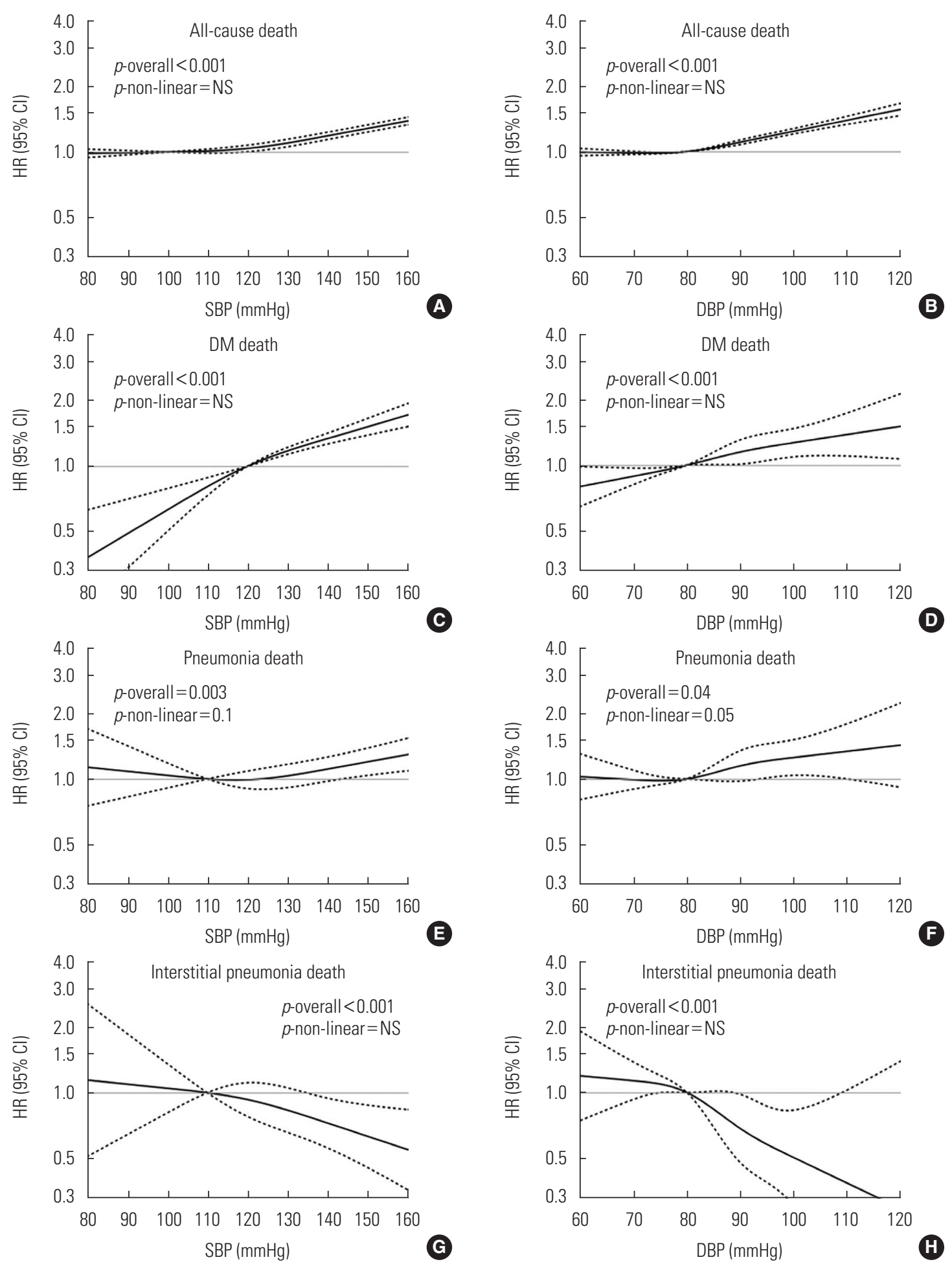

Figure 1. Restricted cubic spline graphs for the risk of all-cause and specific death by SBP (A: all-cause, C: DM, E: pneumonia, G: interstitial pneumonia, I: alcoholic liver disease, K: liver cirrhosis, M: intestinal ischemia, and O: chronic renal failure; 3 knots at 100,120, and $140 \mathrm{mmHg} ; 120 \mathrm{mmHg}$ as a reference) and DBP (B: all-cause, D: DM, F: pneumonia, H: interstitial pneumonia, $\mathrm{J}$ : alcoholic liver disease, L: liver cirrhosis, N: intestinal ischemia, and P: chronic renal failure; 4 knots at 70, 80, 90, 100 mmHg; 80 $\mathrm{mmHg}$ as a reference). Hazard ratios (HRs) and 95\% Confidence intervals (Cis) were estimated in Cox proportional hazard models adjusted for age, sex, body mass index, cigarette smoking, alcohol consumption, physical activity, current anemia, chronic disease score in the participant's medical history, and chronic disease score in the family history. SBP, systolic blood pressure; DBP, diastolic blood pressure; NS, non-specific; DM, diabetes mellitus.

(Continued to the next page) 

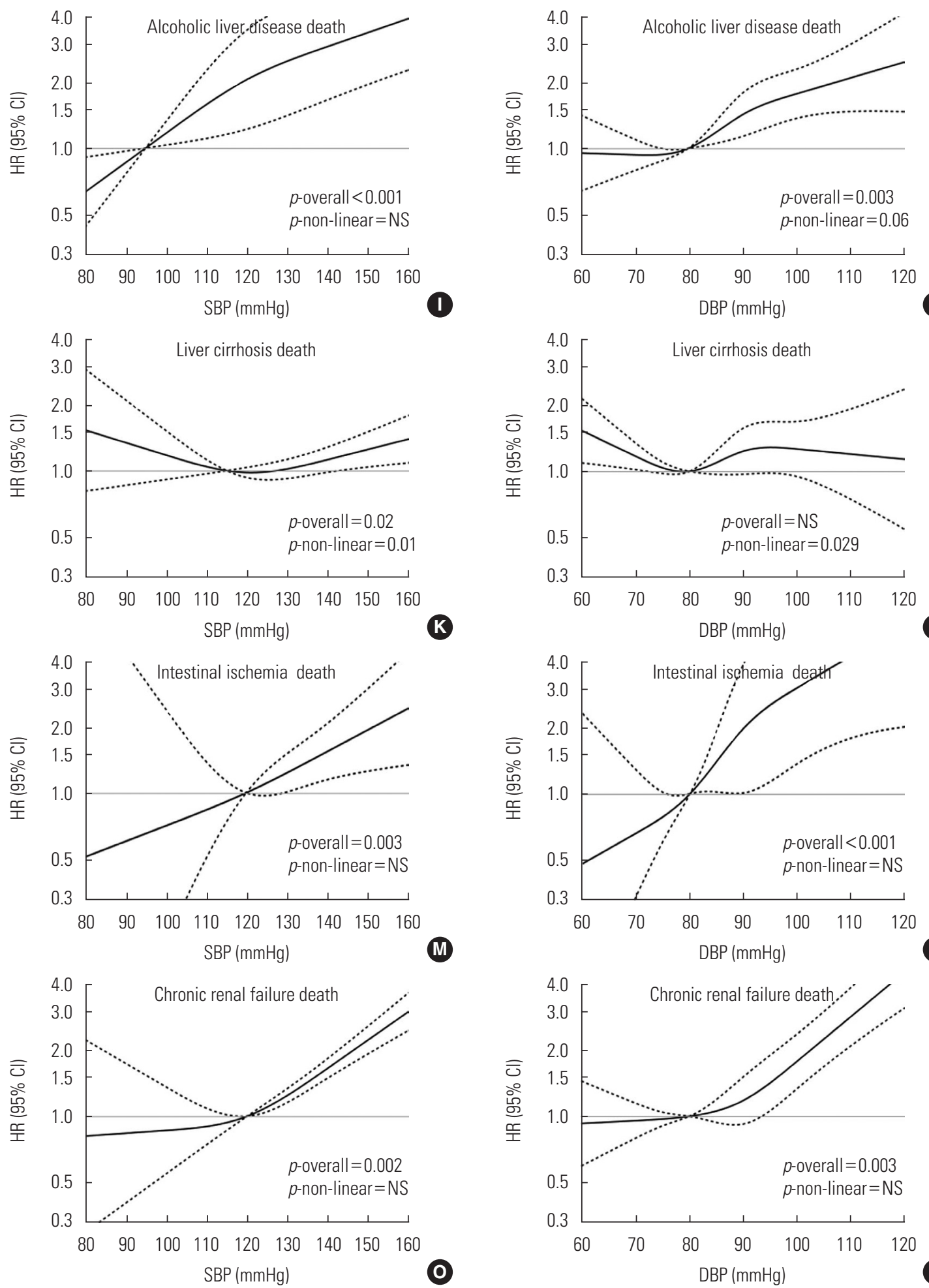

Figure 1. Continued from the previous page.

rollment as the main explanatory variable.

The SBP and DBP levels were classified into 4 groups, as follows: stage II hypertension (SBP $\geq 160$ or DBP $\geq 100 \mathrm{mmHg}$ ), stage I hypertension (SBP 140-159 or DBP 90-99 mmHg), BP

elevation (SBP 120-139 or DBP 80-89 mmHg), and normal BP as a reference category (SBP $<120$ and DBP $<80 \mathrm{mmHg}$ ), following the guidelines of the seventh report of the Joint $\mathrm{Na}$ tional Committee on Prevention, Detection, Evaluation, and 
Treatment of High Blood Pressure [9]. To observe the association of the combined effect of SBP and DBP on the risk of death, we classified each BP variable into a binary variable of hypertension and non-hypertension, and combined them into the following 4 categories: SBP $\geq 140$ and DBP $\geq 90$, SBP $\geq 140$ and DBP $<90$, SBP $<140$ and DBP $\geq 90$, and SBP $<140$ and DBP $<90 \mathrm{mmHg}$.

\section{Statistical Analysis}

Differences in characteristics among different BP groups were evaluated using the chi-square test for categorical variables and 1-way analysis of variance for continuous variables. The associations between BP levels and the risk of death from each cause were assessed in Cox proportional hazards models adjusted for all significant confounders, as shown in Table 1. The confounders adjusted in the Cox proportional hazards models were as follows: age at entry (continuous), sex, smoking status (never vs. ever), alcohol intake (0-1, 2-4, $\geq 5$ times/wk), physical activity (no, rarely, yes), BMI (<18.0, 18.0-22.9, 23.0$29.9, \geq 30.0 \mathrm{~kg} / \mathrm{m}^{2}$ ), chronic disease score in the family history, and chronic disease score in the participant's individual medical history. The chronic disease score in the family history was calculated as the sum of binary scores for a family history of 5 major chronic diseases (hypertension, heart disease, stroke, $\mathrm{DM}$, and cancer) (no=0, yes $=1$ ), and the chronic disease score for each individual participant was calculated as the sum of binary scores for a prior history of 4 major chronic diseases (hypertension, heart disease, stroke, and DM) (no=0, yes $=1)$. Cubic spline figures for the associations between SBP or DBP levels (continuous scales) and the risk of each cause of death were used to examine non-linear effects in each association. When there was a suspicious threshold on the spline graph, we used piecewise linear models to fit non-linearity across all possible thresholds. All statistical analyses were performed using SAS version 9.4 (SAS Institute Inc., Cary, NC, USA).

Table 2. Associations between the categories of SBP and all-cause and specific death in a retrospective cohort enrolled in the Korea National Health Insurance-Health Screening in 2002-2003, followed through 2013

\begin{tabular}{|c|c|c|c|c|c|c|c|c|c|}
\hline & \multirow{3}{*}{$\begin{array}{c}\text { Total } \\
\text { death }(n)\end{array}$} & \multicolumn{7}{|c|}{ SBP (mmHg) } & \multirow{3}{*}{$\begin{array}{l}p \text { for } \\
\text { trend }\end{array}$} \\
\hline & & \multirow{2}{*}{$\frac{<120}{\text { Death }(\mathrm{n})}$} & \multicolumn{2}{|c|}{$120-139$} & \multicolumn{2}{|c|}{$140-159$} & \multicolumn{2}{|r|}{$\geq 160$} & \\
\hline & & & Death (n) & HR $\left(95 \%\right.$ CI) ${ }^{1}$ & Death (n) & HR $(95 \% \mathrm{CI})^{1}$ & Death (n) & HR $(95 \% \text { CI) })^{1}$ & \\
\hline All-cause & 33215 & 6187 & 13256 & $1.09(1.06,1.12)$ & 9527 & $1.22(1.18,1.26)$ & 4245 & $1.46(1.41,1.52)$ & $<0.01$ \\
\hline Tuberculosis & 261 & 57 & 104 & $0.95(0.69,1.31)$ & 66 & $0.91(0.64,1.31)$ & 34 & $1.24(0.80,1.92)$ & 0.58 \\
\hline Hepatitis & 148 & 29 & 68 & $1.38(0.89,2.15)$ & 37 & $1.39(0.84,2.31)$ & 14 & $1.56(0.80,3.03)$ & 0.17 \\
\hline DM, total & 1203 & 146 & 478 & $1.63(1.35,1.96)$ & 382 & $1.83(1.51,2.22)$ & 197 & $2.38(1.91,2.96)$ & $<0.01$ \\
\hline NIDDM & 793 & 102 & 309 & $1.52(1.21,1.91)$ & 256 & $1.77(1.40,2.24)$ & 126 & $2.21(1.69,2.89)$ & $<0.01$ \\
\hline Parkinson disease & 287 & 46 & 134 & $1.36(0.97,1.91)$ & 75 & $1.06(0.73,1.54)$ & 32 & $1.16(0.73,1.84)$ & 0.93 \\
\hline Alzheimer disease & 195 & 37 & 84 & $1.08(0.73,1.60)$ & 44 & $0.76(0.48,1.18)$ & 30 & $1.24(0.76,2.03)$ & 0.9 \\
\hline Pneumonia & 783 & 132 & 310 & $1.12(0.91,1.37)$ & 222 & $1.11(0.89,1.39)$ & 119 & $1.54(1.19,1.98)$ & $<0.01$ \\
\hline Interstitial pneumonia & 187 & 45 & 84 & $0.82(0.57,1.18)$ & 47 & $0.64(0.42,0.98)$ & 11 & $0.38(0.20,0.75)$ & $<0.01$ \\
\hline Aspiration pneumonia & 130 & 24 & 52 & $1.04(0.64,1.70)$ & 32 & $0.90(0.52,1.54)$ & 22 & $1.58(0.87,2.85)$ & 0.33 \\
\hline COPD & 857 & 192 & 341 & $0.91(0.76,1.08)$ & 231 & $0.89(0.73,1.08)$ & 93 & $0.94(0.73,1.21)$ & 0.45 \\
\hline Alcoholic liver disease & 388 & 54 & 181 & $1.88(1.39,2.56)$ & 107 & $2.30(1.64,3.21)$ & 46 & $3.14(2.09,4.72)$ & $<0.01$ \\
\hline Liver cirrhosis & 357 & 74 & 136 & $0.98(0.74,1.31)$ & 102 & $1.23(0.90,1.67)$ & 45 & $1.46(1.00,2.15)$ & 0.02 \\
\hline Intestinal ischemia & 39 & 5 & 18 & $1.81(0.67,4.92)$ & 6 & $0.88(0.26,2.95)$ & 10 & $3.77(1.24,11.40)$ & 0.10 \\
\hline Paralytic ileus except hernia & 41 & 7 & 18 & $1.14(0.47,2.75)$ & 6 & $0.49(0.16,1.47)$ & 10 & $1.91(0.71,5.17)$ & 0.61 \\
\hline Pancreatitis & 49 & 10 & 22 & $1.13(0.53,2.40)$ & 14 & $1.15(0.50,2.66)$ & 3 & $0.68(0.18,2.53)$ & 0.79 \\
\hline Acute pancreatitis & 40 & 7 & 18 & $1.29(0.54,3.12)$ & 12 & $1.38(0.53,3.60)$ & 3 & $0.95(0.24,3.78)$ & 0.85 \\
\hline Renal failure & 378 & 46 & 116 & $1.26(0.89,1.77)$ & 127 & $1.99(1.41,2.81)$ & 89 & $3.60(2.49,5.20)$ & $<0.01$ \\
\hline Chronic renal failure & 294 & 33 & 90 & $1.36(0.91,2.03)$ & 95 & $2.07(1.38,3.10)$ & 76 & $4.27(2.80,6.51)$ & $<0.01$ \\
\hline Acute renal failure & 48 & 9 & 14 & $0.75(0.32,1.74)$ & 16 & $1.23(0.53,2.84)$ & 9 & $1.80(0.69,4.66)$ & 0.11 \\
\hline
\end{tabular}

SBP, systolic blood pressure; HR, hazard ratio; Cl, confidence interval; DM, diabetes mellitus; NIDDM, noninsulin-dependent diabetes mellitus; COPD, chronic obstructive pulmonary disease.

${ }^{1}$ Adjusted for age, sex, body mass index, cigarette smoking, alcohol consumption, and physical activity, current anemia, chronic disease score in the participant's medical history, and chronic disease score in the family history. 


\section{RESULTS}

Of the total subjects, $26.7 \%(n=135360)$ had systolic hypertension ( $\mathrm{SBP} \geq 140 \mathrm{mmHg})$ and $31.3 \%(\mathrm{n}=158579)$ had diastolic hypertension (DBP $\geq 90 \mathrm{mmHg}$ ). Participants in the higher BP groups were older $(p<0.01)$ and had higher serum cholesterol levels than those in the lower BP groups $(p<0.01)$. Both the higher SBP and DBP groups had more subjects with a history of diabetes, heart disease, and stroke (all $p<0.01$ ). However, anemia was more prevalent in the lower SBP and $\mathrm{DBP}$ groups (all $p<0.01$ ). Figure 1 shows the estimated risk for each cause of death in relation to SBP or DBP levels. When adjusted for all potential confounders, the associations of each specific death risk with both SBP and DBP levels were linear, except for LC. Pneumonia had an overall linear effect but a mild non-linear effect ( $p$-non-linearity $=0.03$ for SBP; 0.049 for $D B P)$, and LC had a non-linear effect in the association with SBP and DBP ( $p$-non-linearity $=0.01$ and 0.029 , respectively).
As both SBP and DBP levels increased, the risk of death from $\mathrm{DM}$, pneumonia, ALD, and RF increased steadily ( $p$ for trend $<$ 0.05 for all), while the risk of death from interstitial pneumonia decreased $(p<0.01)$. The risk of $L C$ death was associated with only increasing $\operatorname{SBP}(p=0.02)$, and the risk of death from intestinal ischemia was only associated with increasing DBP $(p<$ 0.01) (Tables 2 and 3 ).

Compared to people with normal SBP, those with stage I systolic hypertension had higher risks for death from DM, ALD, and RF (hazard ratio [HR], 1.83; 95\% Cl, 1.51 to 2.22; $\mathrm{HR}, 2.30$; $95 \% \mathrm{Cl}, 1.64$ to $3.21 ; \mathrm{HR}, 1.99 ; 95 \% \mathrm{Cl}, 1.41$ to 2.81 , respectively) and those with stage II systolic hypertension had much higher risks for death from these causes (HR, 2.38 for DM; HR, 3.14 for ALD, and HR, 3.60 for RF, respectively). Of particular note, the risk of death from DM and ALD increased even with SBP elevation (SBP 120-139 mmHg). The risk of death from pneumonia, LC, and intestinal ischemia was significantly higher in only the stage II systolic hypertension group (HR, 1.54;

Table 3. Associations between the categories of DBP and all-cause and specific death in a retrospective cohort enrolled in the Korea National Health Insurance-Health Screening in 2002-2003, followed through 2013

\begin{tabular}{|c|c|c|c|c|c|c|c|c|c|}
\hline & \multirow{3}{*}{$\begin{array}{c}\text { Total } \\
\text { death (n) }\end{array}$} & \multicolumn{7}{|c|}{ DBP (mmHg) } & \multirow{3}{*}{$\begin{array}{l}p \text { for } \\
\text { trend }\end{array}$} \\
\hline & & \multirow{2}{*}{$\frac{<80}{\text { Death (n) }}$} & \multicolumn{2}{|r|}{$80-89$} & \multicolumn{2}{|r|}{$90-99$} & \multicolumn{2}{|r|}{$\geq 100$} & \\
\hline & & & Death (n) & HR $(95 \% \text { CI })^{1}$ & Death (n) & HR $(95 \% \text { Cl })^{1}$ & Death (n) & HR $\left(95 \%\right.$ CI) ${ }^{1}$ & \\
\hline Tuberculosis & 261 & 101 & 73 & $0.78(0.58,1.06)$ & 59 & $1.07(0.77,1.48)$ & 28 & $1.29(0.84,1.97)$ & 0.26 \\
\hline Hepatitis & 148 & 52 & 46 & $0.94(0.63,1.40)$ & 34 & $1.20(0.77,1.87)$ & 16 & $1.04(0.79,2.49)$ & 0.13 \\
\hline Parkinson disease & 287 & 111 & 94 & $0.87(0.66,1.15)$ & 68 & $1.02(0.75,1.39)$ & 14 & $0.52(0.30,0.91)$ & 0.17 \\
\hline Alzheimer disease & 195 & 64 & 75 & $1.30(0.93,1.81)$ & 45 & $1.29(0.88,1.90)$ & 11 & $0.82(0.43,1.56)$ & 0.77 \\
\hline Pneumonia & 783 & 258 & 272 & $1.12(0.94,1.33)$ & 184 & $1.24(1.03,1.50)$ & 69 & $1.18(0.90,1.55)$ & 0.03 \\
\hline Interstitial pneumonia & 187 & 75 & 72 & $0.91(0.66,1.26)$ & 30 & $0.59(0.39,0.91)$ & 10 & $0.48(0.25,0.93)$ & $<0.01$ \\
\hline Liver cirrhosis & 357 & 127 & 121 & $0.96(0.74,1.23)$ & 74 & $0.96(0.71,1.28)$ & 35 & $1.07(0.73,1.57)$ & 0.84 \\
\hline Intestinal ischemia & 39 & 8 & 9 & $1.20(0.46,3.11)$ & 14 & $3.07(1.27,7.38)$ & 8 & $4.39(1.62,11.88)$ & $<0.01$ \\
\hline Paralytic ileus except hernia & 41 & 14 & 14 & $0.99(0.47,2.09)$ & 6 & $0.66(0.25,1.74)$ & 7 & $1.86(0.74,4.67)$ & 0.46 \\
\hline Pancreatitis & 49 & 18 & 20 & $1.10(0.58,2.09)$ & 7 & $0.63(0.26,1.52)$ & 4 & $0.87(0.29,2.60)$ & 0.44 \\
\hline Acute pancreatitis & 40 & 14 & 18 & $1.25(0.62,2.52)$ & 4 & $0.45(0.15,1.38)$ & 4 & $1.08(0.35,3.34)$ & 0.52 \\
\hline Renal failure & 378 & 115 & 115 & $1.03(0.80,1.34)$ & 90 & $1.31(0.99,1.73)$ & 58 & $2.04(1.48,2.81)$ & $<0.01$ \\
\hline Chronic renal failure & 294 & 91 & 84 & $0.95(0.71,1.28)$ & 73 & $1.33(0.97,1.81)$ & 46 & $2.01(1.40,2.88)$ & $<0.01$ \\
\hline Acute renal failure & 48 & 15 & 18 & $1.21(0.61,2.42)$ & 7 & $0.77(0.31,1.90)$ & 8 & $2.16(0.90,5.17)$ & 0.34 \\
\hline
\end{tabular}

DBP, diastolic blood pressure; HR, hazard ratio; Cl, confidence interval; DM, diabetes mellitus; NIDDM, noninsulin-dependent diabetes mellitus; COPD, chronic obstructive pulmonary disease.

${ }^{1}$ Adjusted for age, sex, body mass index, cigarette smoking, alcohol consumption, and physical activity, current anemia, chronic disease score in the participant's medical history, and chronic disease score in the family history. 
$95 \% \mathrm{Cl}, 1.19$ to $1.98 ; \mathrm{HR}, 1.46 ; 95 \% \mathrm{Cl}, 1.00$ to $2.15 ; \mathrm{HR}, 3.77$; $95 \% \mathrm{Cl}, 1.24$ to 11.40 ). People with stage I systolic hypertension were at a reduced risk for death from interstitial pneumonia (HR, $0.64 ; 95 \% \mathrm{Cl}, 0.42$ to 0.98$)$, and those with stage II systolic hypertension were at a much lower risk $(\mathrm{HR}, 0.38 ; 95 \% \mathrm{Cl}$, 0.20 to 0.75 ) (Table 2).

Compared to people with normal DBP, those with stage I diastolic hypertension had an increased risk for death from DM, ALD, $R F$, and intestinal ischemia $(\mathrm{HR}, 1.24 ; 95 \% \mathrm{Cl}, 1.06$ to 1.46; $\mathrm{HR}, 1.67 ; 95 \% \mathrm{Cl}, 1.27$ to $2.20 ; \mathrm{HR}, 1.31 ; 95 \% \mathrm{Cl}, 0.99$ to 1.73 ; $\mathrm{HR}, 3.07 ; 95 \% \mathrm{Cl}, 1.27$ to 7.38 , respectively). Stage II diastolic hypertension was associated with a much higher risk of death from $D M, A L D, R F$, and intestinal ischemia (HR, 1.42, 2.07, 2.04, 4.39; respectively). The risk of pneumonia death was increased in individuals with stage I diastolic hypertension, but not those with stage II diastolic hypertension. Stage I and II diastolic hypertension were associated with a decreased risk for death from interstitial pneumonia ( $\mathrm{HR}, 0.59 ; 95 \% \mathrm{Cl}, 0.39$ to $0.91 ; \mathrm{HR}, 0.48 ; 95 \% \mathrm{Cl}, 0.25$ to 0.93 ) (Table 3 ).
Compared to the reference group (SBP $<140$ and DBP $<90$ $\mathrm{mmHg}$ ), the presence of only diastolic hypertension increased the risk of death from ALD and intestinal ischemia (HR, 1.46; $95 \% \mathrm{Cl}, 1.03$ to $2.07 ; \mathrm{HR}, 3.61 ; 95 \% \mathrm{Cl}, 1.42$ to 9.20 , respectively). Compared to the reference group, the presence of only systolic hypertension increased the risk of death from DM and $\mathrm{RF}(\mathrm{HR}, 1.39 ; 95 \% \mathrm{Cl}, 1.19$ to $1.63 ; \mathrm{HR}, 2.09 ; 95 \% \mathrm{Cl}, 1.59$ to 2.76 , respectively) compared to both normal SBP and DBP levels, but the risk of death from both diseases was not further increased by the presence of both systolic and diastolic hypertension (HR, 1.39 and 2.17, respectively). Deaths from 2 types of pneumonia (pneumonia and interstitial pneumonia) showed associations with only the presence of both types of hypertension ( $\mathrm{HR}, 1.19 ; 95 \% \mathrm{Cl}, 1.00$ to $1.41 ; \mathrm{HR}, 0.51 ; 95 \% \mathrm{Cl}$, 0.34 to 0.77 , respectively). For the risk of death from LC, the presence of only systolic hypertension without diastolic hypertension was associated with a decreased risk $(\mathrm{HR}, 0.56 ; 95 \% \mathrm{Cl}$, 0.32 to 0.99 ), but the other categories were not associated with the risk of death from $\mathrm{LC}$ (Table 4).

Table 4. The combined effect of SBP and DBP on all-cause and specific death in a retrospective cohort enrolled in the Korea National Health Insurance-Health Screening in 2002-2003, followed by 2013

\begin{tabular}{|c|c|c|c|c|c|}
\hline & $\begin{array}{l}S B P<140, \\
D B P<90\end{array}$ & $\begin{array}{l}\text { SBP }<140, \\
D B P \geq 90\end{array}$ & $\begin{array}{l}\text { SBP } \geq 140 \\
\text { DBP }<90\end{array}$ & $\begin{array}{l}S B P \geq 140, \\
D B P \geq 90\end{array}$ & $p$-value \\
\hline All-cause & 1.00 (reference) & $1.05(1.00,1.10)$ & $1.16(1.12,1.20)$ & $1.25(1.21,1.28)$ & $<0.01$ \\
\hline Tuberculosis & 1.00 (reference) & $1.07(0.63,1.82)$ & $0.75(0.50,1.13)$ & $1.23(0.92,1.64)$ & 0.40 \\
\hline Hepatitis & 1.00 (reference) & $1.60(0.92,2.79)$ & $1.16(0.67,2.01)$ & $1.24(0.82,1.86)$ & 0.28 \\
\hline DM, total & 1.00 (reference) & $1.06(0.82,1.38)$ & $1.39(1.19,1.63)$ & $1.39(1.21,1.59)$ & $<0.01$ \\
\hline NIDDM & 1.00 (reference) & $1.08(0.78,1.49)$ & $1.42(1.17,1.73)$ & $1.39(1.18,1.65)$ & $<0.01$ \\
\hline Parkinson diseases & 1.00 (reference) & $0.91(0.54,1.55)$ & $0.81(0.57,1.15)$ & $0.90(0.67,1.20)$ & 0.31 \\
\hline Alzheimer disease & 1.00 (reference) & $1.31(0.72,2.38)$ & $0.82(0.55,1.24)$ & $0.90(0.63,1.29)$ & 0.42 \\
\hline Pneumonia & 1.00 (reference) & $1.20(0.88,1.63)$ & $1.12(0.91,1.36)$ & $1.19(1.00,1.41)$ & 0.05 \\
\hline Interstitial pneumonia & 1.00 (reference) & $0.82(0.44,1.53)$ & $0.88(0.58,1.33)$ & $0.51(0.34,0.77)$ & $<0.01$ \\
\hline Aspiration pneumonia & 1.00 (reference) & $1.10(0.50,2.40)$ & $0.77(0.44,1.33)$ & $1.26(0.84,1.89)$ & 0.47 \\
\hline COPD & 1.00 (reference) & $1.01(0.74,1.37)$ & $0.84(0.68,1.03)$ & $1.05(0.89,1.24)$ & 0.98 \\
\hline Alcoholic liver disease & 1.00 (reference) & $1.46(1.03,2.07)$ & $1.19(0.81,1.74)$ & $1.83(1.45,2.31)$ & $<0.01$ \\
\hline Liver cirrhosis & 1.00 (reference) & $0.56(0.32,0.99)$ & $1.28(0.94,1.76)$ & $1.22(0.95,1.57)$ & 0.07 \\
\hline Intestinal ischemia & 1.00 (reference) & $3.61(1.42,9.20)$ & - & $2.15(1.07,4.33)$ & 0.14 \\
\hline Paralytic ileus except hernia & 1.00 (reference) & $0.89(0.21,3.78)$ & $0.64(0.24,1.70)$ & $0.92(0.44,1.92)$ & 0.66 \\
\hline Pancreatitis & 1.00 (reference) & $0.88(0.27,2.91)$ & $1.45(0.67,3.12)$ & $0.67(0.30,1.49)$ & 0.57 \\
\hline Acute pancreatitis & 1.00 (reference) & $0.35(0.05,2.58)$ & $1.54(0.67,3.52)$ & $0.70(0.30,1.65)$ & 0.68 \\
\hline Renal failure & 1.00 (reference) & $1.06(0.63,1.79)$ & $2.09(1.59,2.76)$ & $2.06(1.62,2.62)$ & $<0.01$ \\
\hline Chronic renal failure & 1.00 (reference) & $1.14(0.64,2.02)$ & $2.15(1.57,2.94)$ & $2.17(1.65,2.85)$ & $<0.01$ \\
\hline Acute renal failure & 1.00 (reference) & $0.44(0.06,3.30)$ & $1.83(0.87,3.83)$ & $1.44(0.73,2.85)$ & 0.17 \\
\hline
\end{tabular}

Values are presented as hazard ratio (95\% confidence interval).

Hazard ratios were adjusted for age, sex, body mass index, cigarette smoking, alcohol consumption, physical activity, current anemia, chronic disease score in the participant's medical history, and chronic disease score in the family history.

SBP, systolic blood pressure; DBP, diastolic blood pressure; DM, diabetes mellitus; NIDDM, noninsulin-dependent diabetes mellitus; COPD, chronic obstructive pulmonary disease. 


\section{DISCUSSION}

In our study, as BP levels increased, the risk of death from DM, pneumonia, ALD, intestinal ischemia, and RF increased linearly, while the risk of death from interstitial pneumonia decreased linearly. The risk of LC death showed a non-linear association with BP levels. Stage I systolic or diastolic hypertension was associated with an increased risk of death from DM, ALD, and $\mathrm{RF}$, relative to the corresponding normal $\mathrm{BP}$ levels, but was associated with a decreased risk of death from interstitial pneumonia. Stage II systolic and diastolic hypertension were associated with a much higher risk of death from DM, ALD, and RF. The risk of death from pneumonia and LC was only elevated in participants with stage II systolic hypertension. Death from intestinal ischemia was associated with stage II systolic hypertension and stage I and II diastolic hypertension. In the assessment of the combined risk of SBP and DBP for death, the risk of death from DM and RF increased with systolic hypertension alone, but the risk of death from ALD and intestinal ischemia was increased only by diastolic hypertension. The risk of death from pneumonia and interstitial pneumonia showed an association with the comorbidity of both systolic and diastolic hypertension.

Hypertension and DM are known to involve the same vascular complications and target organs $[10,11]$. Insulin resistance is the key feature of the pathogenesis of DM, and is also known to be associated with hypertension by several mechanisms that are closely related to vascular wall activity [12]. Compared with the general population, individuals with hypertension are more likely to have DM, and those with DM are also more likely to have hypertension $[10,11]$. In Korea, only $25.9 \%$ of patients with hypertension have diabetes, but $50 \%$ of patients with diabetes have hypertension $[13,14]$. In the latter group, diabetes may have been diagnosed earlier than hypertension, and diabetes may be recognized as the primary cause of death in the death certificate data. Therefore, we further analyzed our data to eliminate this possibility after excluding subjects with a past history of DM. In the results of the restricted analysis, which only included subjects with no past history of DM, individuals with systolic or diastolic hypertension had a higher risk of diabetes death than their counterparts with normal BP (Table S2).

Long-term persistence of hypertension leads to renal damage, resulting in chronic renal disease [15]. Secondary hypertension may also occur as a sequela of chronic renal disease, even if hypertension does not occur first [15]. The increased risk of $\mathrm{RF}$ in hypertension patients is biologically plausible.

It is known that malignant systemic hypertension can aggravate underlying chronic kidney disease (CKD) rapidly. Uncontrolled high BP can cause damage to arteries around the kidney, which leads to decreased blood flow to kidney tissue $[15,16]$. However, controversy has long existed regarding whether mild-to-moderate elevation in systemic BP often triggers CKD [17]. Recent studies have showed that high-risk genes, such as APOL 1 in the African-American population, are associated with both focal segmental glomerulosclerosis and secondary hypertension $[18,19]$. Further studies of genetic markers are required to reveal the underlying mechanism.

In our study, an increased risk of death from ALD was prominently found in individuals with high DBP, with a dose-dependent trend. In Korea, hypertension is known to be related to alcohol consumption patterns [20], which can affect heart and cardiac end-diastolic pressure. Patients with alcoholic fatty liver often present with increased BP [21]. We suggest that ALD and hypertension share the common risk factor of alcohol, which may increase the risk of ALD mortality in hypertension patients, in particular those with diastolic hypertension, compared to people with low BP levels. Contrarily, the hemodynamic characteristics of $L C$ involve vasodilation, which leads to low arterial BP [21]. It can be expected that low BP levels result from the hemodynamics of LC. If blood vessels function properly, arterial hypertension may occur in patients with early LC according to the natural history of LC, but BP falls to normal levels as LC progresses [22]. However, about $15 \%$ of LC patients are reported to have hypertension due to alteration of vasodilatation and vasoconstriction through dysfunctions in the circulatory system [21]. Patients with bleeding tendency due to end-stage $\mathrm{LC}$ are at a higher risk of fatal bleeding. It is possible that, in our study, high baseline SBP increased the future risk of vascular rupture. The decreased risk in the high DBP-only group could also be explained in terms of fatal bleeding. The lowest risk of death from LC was found in individuals with high DBP and normal SBP, which corresponds to a considerably low pulse pressure, a good predictor for stroke and other vascular accidents [23]. The spline graph between LC mortality and BP showed a non-linear association, suggesting that complex hemodynamic characteristics affect the relationship between LC and hypertension. In this study, we did not observe a relationship between BP levels in LC patients and mortality risk after LC diagnosis, it is necessary to further 
examine the relationship between BP levels after LC diagnosis and the risk of death.

Although a direct relationship between pneumonia and essential hypertension has not been well established [24], there might be an indirect relationship. The incidence of pneumonia in the acute stage after stroke diagnosis is common as an acute complication of stroke and silent infarction, leading to sudden death [25-27]. Unlike pneumonia, no report indicating that high BP increased the risk of mortality from interstitial pneumonia was found in the literature. The lower risk of death from interstitial pneumonia observed in our cohort in individuals with hypertension may have been a coincidence, and this possibility should be investigated in larger cohort studies.

Intestinal ischemia is associated with acute or chronic low blood flow to the gut due to atherosclerosis, cardiac arrhythmias and other reasons. Although arterial hypertension is not a direct risk factor for intestinal ischemia in terms of gastrointestinal vascular pathology, several experimental results have shown that blood vessels in hypertensive animals are more sensitive to vasoconstrictors [28]. These results suggest that hypertension may induce intestinal ischemia and increase the risk of death in the presence of any stress conditions, although it is not a common mechanism of intestinal ischemia. In this study, this risk was more closely related to DBP than SBP. Diastolic hypertension seems to have an indirect link with intestinal ischemia because of its association with aortic aneurysm [29].

There are several limitations of this study. First, the causes of death from national death records were not verified, but information on the death certificate is known to be reasonably valid in comparison with medical records [30]. Second, the method of BP measurement may have varied among different healthcare centers, leading to measurement error. Third, potential residual confounders such as compliance with antihypertensive medication or other drugs prescribed for the comorbidities of hypertension could not be considered. For antihypertensive medicines, we constructed the variables using drug claim information and included the variables in the multivariate model. As a result, we did not obtain an HR value due to an abnormal proportion of non-users (almost 5\%) and rare events from certain diseases. Fourth, this study did not consider the influence of other important factors such as comorbidities of hypertension and compliance on death. Fifth, the possible misclassification of intestinal ischemia (vascular disorder of intestine) should be kept in mind, due to recent changes in the newly introduced ICD-10, which integrated several diseases into 2 different categories. Sixth, we could not find evidence that the measurement process of BP was standardized, so misclassification error should be considered. The prospective design, relatively large number of participants, sufficient duration of follow-up, completeness of the follow-up data, and the inclusion of ethnically homogeneous participants could be considered as strengths of this study.

Hypertension is a major concern for Korean public health practitioners due to its large disease burden [31,32], but its consequences are usually understood as limited to vascular complications. However, in this study, we investigated associations between BP levels and the risk of death from other specific diseases. We expect that the results of our study will serve as evidence that researchers should include more disease entities when estimating the disease burden of hypertension. Our findings suggest that patients with hypertension are at an elevated risk of death from a variety of additional diseases. These findings show that researchers and public health practitioners may need to track more diseases related to hypertension for patient care, the estimation of disease burden, and policymaking.

In conclusion, Koreans with systolic or diastolic hypertension showed an increased risk of death from DM, ALD, and RF, but a decreased risk of death from interstitial pneumonia. The risk of death from pneumonia and LC was only linked to stage II systolic hypertension. Intestinal ischemia was associated with diastolic hypertension and stage II systolic hypertension. Increased BP seems to increase the risk of death from certain diseases other than cerebrocardiovascular diseases, suggesting that arterial hypertension may affect several organs, in addition to its well-known target organs, although the mechanism remains unclear.

\section{ACKNOWLEDGEMENTS}

This research was supported by the Korea Health Technology R\&D Project through the Korea Health Industry Development Institute (KHIDI), the Ministry of Health \& Welfare, Republic of Korea (HI16C1127), and Seoul National University Hospital (2018).

This study used NHIS-HEALS data (NHIS-2018-2-235) developed by the National Health Insurance Service (NHIS), which we gratefully acknowledge. 


\section{CONFLICT OF INTEREST}

The authors have no conflicts of interest associated with the material presented in this paper.

\section{SUPPLEMENTAL MATERIALS}

Supplementary Materials 1-5: Table S1-S5 are available at https://www.jpmph.org/.

\section{ORCID}

Jeoungbin Choi http://orcid.org/0000-0001-7655-6273

Jieun Jang http://orcid.org/0000-0001-6970-9374

Yoonsuk An http://orcid.org/0000-0002-2499-2281

Sue K. Park http://orcid.org/0000-0001-5002-9707

\section{REFERENCES}

1. Bahk YY, Park YK, Na BK, Sohn WM, Hong SJ, Chai JY, et al. Survey on intestinal helminthic infection status of students in two counties, Hadong-gun and Goseong-gun, Korea. Korean J Parasitol 2018;56(4):335-339.

2. Nicholls MG. Hypertension, hypertrophy, heart failure. Heart 1996;76(3 Suppl 3):92-97.

3. MacMahon S, Peto R, Cutler J, Collins R, Sorlie P, Neaton J, et al. Blood pressure, stroke, and coronary heart disease. Part 1, prolonged differences in blood pressure: prospective observational studies corrected for the regression dilution bias. Lancet 1990;335(8692):765-774.

4. Alexander RW. Hypertension and the pathogenesis of atherosclerosis. Oxidative stress and the mediation of arterial inflammatory response: a new perspective. Hypertension 1995;25(2): 155-161.

5. Mensah GA, Croft JB, Giles WH. The heart, kidney, and brain as target organs in hypertension. Curr Probl Cardiol 2003;28(2): 156-193.

6. Seong SC, Kim YY, Park SK, Khang YH, Kim HC, Park JH, et al. Cohort profile: the National Health Insurance Service-National Health Screening Cohort (NHIS-HEALS) in Korea. BMJ Open 2017;7(9):e016640.

7. Göbel BO, Schulte-Göbel A, Weisser B, Glänzer K, Vetter H, Düsing R. Arterial blood pressure. Correlation with erythrocyte count, hematocrit, and hemoglobin concentration. Am J Hypertens 1991;4(1 Pt 1):14-19.
8. Gilbertson DT, Ebben JP, Foley RN, Weinhandl ED, Bradbury BD, Collins AJ. Hemoglobin level variability: associations with mortality. Clin J Am Soc Nephrol 2008;3(1):133-138.

9. Chobanian AV, Bakris GL, Black HR, Cushman WC, Green LA, Izzo JL Jr, et al. Seventh report of the Joint National Committee on Prevention, Detection, Evaluation, and Treatment of High Blood Pressure. Hypertension 2003;42(6):1206-1252.

10. Long AN, Dagogo-Jack S. Comorbidities of diabetes and hypertension: mechanisms and approach to target organ protection. J Clin Hypertens (Greenwich) 2011;13(4):244-251.

11. Khosravi A, Emamian MH, Hashemi H, Fotouhi A. Pre-hypertension and the risk of diabetes mellitus incidence using a marginal structural model in an Iranian prospective cohort study. Epidemiol Health 2018;40:e2018026.

12. Morales-Villegas E. Dyslipidemia, hypertension and diabetes metaflammation: a unique mechanism for 3 risk factors. Curr Hypertens Rev 2013;9(4):278-296.

13. Centers for Disease Control and Prevention. National diabetes fact sheet, 2011 [cited 2018 Sep 3]. Available from: https:// www.cdc.gov/diabetes/pubs/pdf/ndfs_2011.pdf.

14. Korean Diabetes Association. Diabetes fact sheet in Korea 2016 [cited 2018 Sep 3]. Available at: http://www.diabetes.or.kr/ temp/KDA_fact_sheet\%202016.pdf.

15. Gargiulo R, Suhail F, Lerma EV. Hypertension and chronic kidney disease. Dis Mon 2015;61(9):387-395.

16. Hill GS. Hypertensive nephrosclerosis. Curr Opin Nephrol Hypertens 2008;17(3):266-270.

17. Freedman $\mathrm{BI}$, Cohen $\mathrm{AH}$. Hypertension-attributed nephropathy: what's in a name? Nat Rev Nephrol 2016;12(1):27-36.

18. Freedman BI, Iskandar SS, Appel RG. The link between hypertension and nephrosclerosis. Am J Kidney Dis 1995;25(2):207221.

19. Meyrier A. Nephrosclerosis: a term in quest of a disease. Nephron 2015;129(4):276-282.

20. Hong SW, Linton JA, Shim JY, Lee HR, Kang HT. Association of alcohol consumption pattern with risk of hypertension in Korean adults based on the 2010-2012 KNHANES. Alcohol 2016; 54:17-22.

21. Henriksen JH, Moller S. Liver cirrhosis and arterial hypertension. World J Gastroenterol 2006;12(5):678-685.

22. Schwartz DT. The relation of cirrhosis of the liver to renal hypertension. A review of 639 autopsied cases. Ann Intern Med 1967;66(5):862-869.

23. Dart AM, Kingwell BA. Pulse pressure--a review of mechanisms and clinical relevance. J Am Coll Cardiol 2001;37(4):975-984. 
24. Shen Y, Tian Z, Lu D, Huang J, Zhang Z, Li X, et al. Impact of pneumonia and lung cancer on mortality of women with hypertension. Sci Rep 2016;6(1):20.

25. Nakagawa T, Sekizawa K, Nakajoh K, Tanji H, Arai H, Sasaki H. Silent cerebral infarction: a potential risk for pneumonia in the elderly. J Intern Med 2000;247(2):255-259.

26. Okuro M, Okuno T, Himeno T, Morita T, Iritani $\mathrm{O}$, Yano $\mathrm{H}$, et al. Severe hypertension on admission and acute pneumonia in the elderly with acute cerebral hemorrhage. Innov Aging 2017; 1(Suppl 1):300-301.

27. Ishigami K, Okuro M, Koizumi Y, Satoh K, Iritani O, Yano H, et al. Association of severe hypertension with pneumonia in elderly patients with acute ischemic stroke. Hypertens Res 2012; 35(6):648-653.

28. Gallavan RH Jr, Parks DA, Jacobson ED. Pathophysiology of gastrointestinal circulation; 2011 [cited 2018 Sep 3]. Available from: https://onlinelibrary.wiley.com/doi/pdf/10.1002/cphy. cp060147.

29. Tsialtas D, Bolognesi MG, Volpi R, Bolognesi R. Atherosclerotic vascular diseases have really the same risk factors? Comparison between large abdominal aortic aneurysm and obstructive non-coronary arterial disease. Vascular 2017;25(6):629-634.

30. Won TY, Kang BS, Im TH, Choi HJ. The study of accuracy of death statistics. J Korean Soc Emerg Med 2007;18(3):256-262 (Korean).

31. Kim HC, Oh SM. Noncommunicable diseases: current status of major modifiable risk factors in Korea. J Prev Med Public Health 2013;46(4):165-172.

32. Lee JH, Yang DH, Park HS, Cho Y, Jun JE, Park WH, et al. Incidence of hypertension in Korea: 5-year follow-up study. J Korean Med Sci 2011;26(10):1286-1292. 